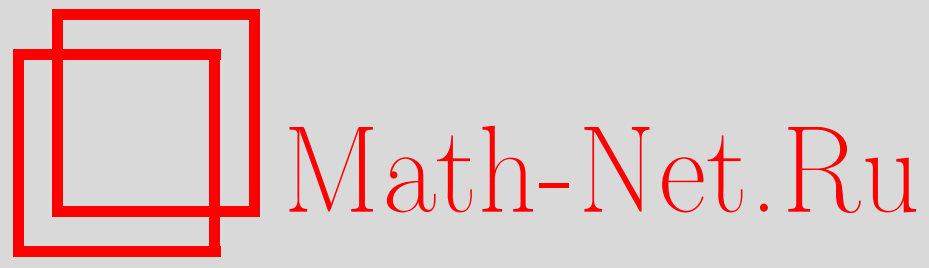

А. Н. Старцев, Об одной модели с взаимодействием частиц двух типов, обобщающей процесс эпидемии Бартлетта-Мак-Кендрика, Теория вероятн. и ее примен., 2001, том 46, выпуск 3, 463-482

DOI: https://doi.org/10.4213/tvp3896

Использование Общероссийского математического портала MathNet.Ru подразумевает, что вы прочитали и согласны с пользовательским соглашением

http://www.mathnet.ru/rus/agreement

Параметры загрузки:

IP: 54.174 .149 .18

26 апреля 2023 г., 18:31:34

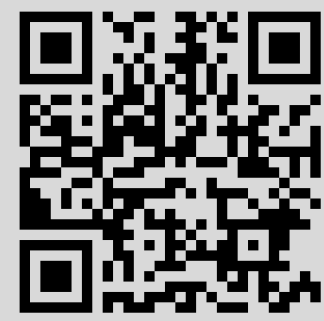




\title{
ОБ ОДНОЙ МОДЕЛИ С ВЗАИМОДЕЙСТВИЕМ ЧАСТИЦ ДВУХ ТИПОВ, ОБОБЩАЮЩЕЙ ПРОЦЕСС ЭПИДЕМИИ БАРТЛЕТТА-МАК-КЕНДРИКА
}

\begin{abstract}
Рассматривается замкнутая (по числу частиц) система взаимодействующих частиц двух типов $A$ и $B$. Частицы типа $B$ обладают запасом некоторой «энергии», а частицы типа $A$ способны приобретать ее при взаимодействии с единичной интенсивностью, обладая при этом некоторым порогом чувствительности. Если запас приобретенной «энергии» достигает уровня чувствительности, то частица типа $A$ переходит в частицу типа $B$. Частица типа $B$, исчерпавшая запас своей «энергии», погибает. Процесс заканчивается, если в системе остаются частицы одного типа. При условиях, когда в системе достаточно много частиц обоих типов, описывается класс предельных законов для числа частиц $\nu$, изменивших свой тип в предположении, что уровни чувствительности частиц типа $A$ задаются независимыми показательно распределенными случайными величинами с параметром 1 , а времена потери «энергии》 частицами типа $B$ - произвольными одинаково распределенными взаимно независимыми случайными величинами, не зависящими от предыдущих случайных величин.
\end{abstract}

Ключевые слова и фразы: частицы, взаимодействие, изменение типа, немарковские модели, порядковые статистики, граничные задачи, предельные теоремы.

1. Введение. Рассматривается замкнутая (по числу частиц) система взаимодействующих частиц двух типов $A$ и $B$. Частицы типа $B$ обладают запасом некоторой «энергии», а частицы типа $A$ способны приобретать ее при взаимодействии с единичной интенсивностью, обладая при этом некоторым порогом чувствительности. Если запас приобретенной «энергии» достигает уровня чувствительности, то частица типа $A$ переходит в частицу типа $B$. Частица типа $B$, исчерпавшая запас своей «энергии», погибает. Процесс заканчивается, если в системе остаются частицы одного типа. Пусть в момент времени $t>0$ система состоит из $\xi_{A}(t)$ частиц типа $A$ и $\xi_{B}(t)$ частиц типа $B$, причем $\xi_{A}(0)=n$, $\xi_{B}(0)=m$. Далее, пусть времена потери «энергии» частицами типа $B$

\footnotetext{
${ }^{*}$ Институт математики АН РУ, ул. Ф. Ходжаева, 29, 700143 Ташкент, Узбекистан; e-mail: root@im.tashkent.su
} 
задаются независимыми одинаково распределенными случайными величинами (н.о.р.с.в.) $T_{1}, \ldots, T_{m}, \ldots$, а уровни чувствительности частиц типа $B$ - н.с.в. $L_{1}, \ldots, L_{n}$, имеющими показательное распределение с параметром $1\left(L_{i} \in \exp (1)\right)$ и не зависящими от $T_{1}, \ldots, T_{m}$.

Легко показать, что описанный выше процесс в общем случае не будет марковским. Однако если распределение $F(x)=\mathbf{P}\left\{T_{1}<x\right\}$ абсолютно непрерывно, то $\xi(t)=\left(\xi_{A}(t), \xi_{B}(t)\right)$ будет неоднородным во времени марковским процессом с фазовым пространством $\mathbf{Z}_{+}^{2}$, с поглощающими состояниями вида $(k, 0)$ и следующими вероятностями возможных переходов:

$$
\left\{\begin{array}{l}
\mathbf{P}\{\xi(t+\Delta t)=(s-1, i+1) \mid \xi(t)=(s, i)\}=s i \Delta t+o(\Delta t), \\
\mathbf{P}\{\xi(t+\Delta t)=(s, i-1) \mid \xi(t)=(s, i)\}=\alpha(t) i \Delta t+o(\Delta t), \\
\mathbf{P}\{\xi(t+\Delta t)=(s, i) \mid \xi(t)=(s, i)\}=1-(\alpha(t)+s) i \Delta t+o(\Delta t),
\end{array}\right.
$$

где $\alpha(t)=F^{\prime}(t) /(1-F(t))$.

Действительно, если $(s, i)$ - состояние процесса в некоторый момент $t>0$, а $\eta_{t}$ - количество «энергии», приобретенное каждой из $s$ существующих частиц типа $A$ к этому моменту, то вероятность того, что $j$-я из них не изменит типа в интервале $(t, t+\Delta t)$, равна

$$
\begin{aligned}
& \mathbf{P}\left\{L_{j}>\eta_{t}+i \Delta t \mid L_{j}>\eta_{t}\right\} \\
& \quad=\int_{0}^{\infty} \mathbf{P}\left\{L_{j}>x+i \Delta t \mid L_{j}>x\right\} d \mathbf{P}\left\{\eta_{t}<x\right\}=e^{-i \Delta t}
\end{aligned}
$$

а вероятность того, что $k$-я из $i$ существующих частиц типа $B$ погибнет в том же интервале, равна

$$
\mathbf{P}\left\{T_{k}>t+\Delta t \mid T_{k}>t\right\}=\frac{F(t+\Delta t)-F(t)}{1-F(t)}=\alpha(t) \Delta t+o(\Delta t),
$$

откуда уже легко находятся вероятности переходов (1.1).

Заметим, что формализация исходной модели как полумарковского процесса приводит к большим трудностям ввиду сложности в описании времен пребывания процесса в своих состояниях.

Если $T_{1}, T_{2}, \ldots$ также показательно распределены с параметром $\rho$, т.е. $F(x)=1-\exp (-\rho x)$, то $\alpha(t)=\rho$ и мы приходим к однородному марковскому процессу со следующими вероятностями возможных переходов:

$$
\left\{\begin{array}{l}
\mathbf{P}\{\xi(t+\Delta t)=(s-1, i+1) \mid \xi(t)=(s, i)\}=s i \Delta t+o(\Delta t), \\
\mathbf{P}\{\xi(t+\Delta t)=(s, i-1) \mid \xi(t)=(s, i)\}=\rho i \Delta t+o(\Delta t) \\
\mathbf{P}\{\xi(t+\Delta t)=(s, i) \mid \xi(t)=(s, i)\}=1-(\rho+s) i \Delta t+o(\Delta t) .
\end{array}\right.
$$


Модель (1.2) известна как процесс Бартлетта - Мак-Кендрика и достаточно хорошо изучена как модель распространения эпидемий. (Обзор результатов и литературные ссылки по этому поводу см. в работе автора [1].) В этом случае $\xi_{A}(t)$ и $\xi_{B}(t)$ интерпретируются как число восприимчивых индивидуумов в популяции и число источников инфекции соответственно, а величины $T_{1}, T_{2}, \ldots$ и $L_{1}, L_{2}, \ldots$-соответственно, как инфекционные периоды источников инфекции и иммунологические барьеры восприимчивых (см. [2]). При этом первый из переходов в (1.2) отражает процесс заражения восприимчивых, второй - процесс устранения источников инфекции, а функционал $\nu$ - число частиц, изменивших свой тип, - интерпретируется как размер эпидемии.

Более общие, в сравнении с (1.2), марковские модели с взаимодействием частиц были введены Б. А. Севастьяновым [3], и для них были получены соответствующие уравнения Колмогорова для производящей функции вероятностей состояний, которые, однако, в общем случае не разрешимы в явном виде. Тем не менее, ряд существенных результатов точного и асимптотического характера был получен в работах А. В. Калинкина. В частности, в работе [4] процессы эпидемий исследуются прямым решением уравнений Колмогорова.

Объектом данного исследования является число частиц $\nu$, изменивших свой тип к моменту окончания процесса, для распределения которого можно записать следующее представление:

$$
\mathbf{P}\{\nu=k\}=\mathbf{P}\left\{L_{(1)} \leqslant S_{m}, \ldots, L_{(k)} \leqslant S_{m+k-1}, L_{(k+1)}>S_{m+k}\right\},
$$

где $L_{(1)} \leqslant L_{(2)} \leqslant \cdots \leqslant L_{(n)}$ - вариационный ряд, образованный из с.в. $L_{1}, L_{2}, \ldots, L_{n}$, a $S_{k}=T_{1}+T_{2}+\cdots+T_{k}$.

Соотношение (1.3) легко следует из определения исходного процесса взаимодействия. Действительно, величина $S_{m}$ представляет собой суммарную «энергию», которую приобретают частицы типа $A$ от $m$ первоначальных частиц типа $B$, и если при этом $L_{(1)}=\min \left(L_{1}, \ldots, L_{n}\right)>$ $S_{m}$, то ни одна из частиц типа $A$ не сможет изменить свой тип, т.е. $\mathbf{P}\{\nu=0\}=\mathbf{P}\left\{L_{(1)}>S_{m}\right\}$. Дальнейшие рассуждения проводятся аналогично и приводят к (1.3).

Отметим, что Болл [5] получил ряд результатов точного и асимптотического характера для рассматриваемого процесса, но в терминах процесса эпидемий (обзор по этому поводу см. в [1]), при этом он также основывался на соотношении (1.3), которое впервые было предложено Селлке [6], но в ситуации, когда как $\left\{T_{i}\right\}$, так и $\left\{L_{i}\right\}$ показательно распределены (комментарии по этому поводу см. также в работе [1]).

Работа автора [1], посвященная процессам эпидемий, соответствует случаю, когда частица типа $A$, получившая запас «энергии», превосходящий ее уровень чувствительности, также погибает. При этом величина $\nu$ задает число частиц типа $A$, погибших к моменту поглощения 
процесса, и в этом случае будет иметь место более простое, чем (1.3), соотношение

$$
\mathbf{P}\{\nu=k\}=\mathbf{P}\left\{L_{(k)} \leqslant S_{m}, L_{(k+1)}>S_{m}\right\}
$$

которое позволяет описать класс предельных распределений для $\nu$ при более общих предположениях относительно $\left\{T_{i}\right\}$ и $\left\{L_{i}\right\}$, основываясь на асимптотической теории порядковых статистик.

Если же исходить из (1.3), то возникает необходимость в асимптотической теории для совместного распределения порядковых статистик $L_{(1)}, L_{(2)}, \ldots, L_{(k)}$ при различных соотношениях между $k$ и $n$. Соответствующая теория пока отсутствует и ее развитие упирается в большие аналитические трудности. Однако если $L_{1}, L_{2}, \ldots, L_{n}-$ н.о.р.с.в. с показательным распределением, а $T_{1}, T_{2}, \ldots$ - н.о.р.с.в., не зависящие от $L_{1}, L_{2}, \ldots, L_{n}$, то задачу о распределении величины $\nu$ удается свести к задаче о моменте первого выхода через подвижную границу сумм н.с.в. в схеме серий.

Аналогичный подход для асимптотического анализа модели (1.2) был использован в работе А. В. Нагаева и автора [7]. В данной статье эти результаты существенно обобщаются, а применяемая в ней редукция к граничным задачам является принципиально иной. Бо́льшая общность рассматриваемой модели состоит также и в том, что в ней появляются новые параметры, участвующие в описании предельных случаев, к их числу относится, прежде всего, дисперсия с.в. $T_{1}$.

Отметим также, что модели рассматриваемого типа могут служить и для описания реакции химической кинетики и при этом величины $L_{1}, L_{2}, \ldots, L_{n}$ интерпретируются как потенциальные барьеры молекул [8].

2. Редукция к граничной задаче. Известно $[9$, гл. 1 , п. 6], что если $L_{1}, L_{2}, \ldots, L_{n}-$ н.о.p.с.в. и $L_{1} \in \exp (1)$, то $L_{(1)}, L_{(2)}-L_{(1)}, \ldots$, $L_{(n)}-L_{(n-1)}$ являются н.с.в., причем $L_{(k+1)}-L_{(k)} \in \exp (n-k)$. А тогда (1.3) может быть записано в виде

$$
\mathbf{P}\{\nu=k\}=\mathbf{P}\left\{\widetilde{S}_{1} \leqslant S_{m}, \ldots, \widetilde{S}_{k} \leqslant S_{m+k-1}, \widetilde{S}_{k+1}>S_{m+k}\right\}
$$

где $\widetilde{S}_{k}=\tilde{\xi}_{1}+\cdots+\tilde{\xi}_{k}, \tilde{\xi}_{i}=\xi_{i} /(n-i+1)$, а $\xi_{1}, \xi_{2}, \ldots, \xi_{n}-$ н.о.р.с.в. такие, что $\xi_{1} \in \exp (1)$.

Если ввести обозначения $\eta_{1}=\tilde{\xi}_{1}, \eta_{2}=\tilde{\xi}_{2}-T_{m+1}, \ldots, \eta_{k+1}=\tilde{\xi}_{k+1}-$ $T_{m+k}, \zeta_{k}=\eta_{1}+\cdots+\eta_{k}$, то соотношение $(2.1)$ перепишется в виде

$$
\mathbf{P}\{\nu=k\}=\mathbf{P}\left\{\zeta_{1} \leqslant S_{m}, \ldots, \zeta_{k} \leqslant S_{m}, \zeta_{k+1}>S_{m}\right\} .
$$

Как уже отмечалось, асимптотический анализ распределения $\nu$ будет проводится при $n \rightarrow \infty$ в схеме серий. Теперь введем следующие 
обозначения:

$$
\begin{gathered}
A_{k} \equiv A_{k}(n)=\mathbf{E} S_{k}, \quad B_{k}^{2} \equiv B_{k}^{2}(n)=\mathbf{D} \zeta_{k}, \quad C_{k} \equiv C_{k}(n)=\mathbf{E} \zeta_{k+1}, \\
D_{n}^{2}=\mathbf{D} \zeta_{k_{n}+1}, \quad t_{k}=t_{n k}=\frac{\mathbf{D} \zeta_{k}}{D_{n}^{2}}, \quad k=1, \ldots, k_{n}, \\
\Pi_{k}(\lambda)=\frac{\lambda^{k}}{k !} e^{-\lambda}, \quad \Phi(x)=\frac{1}{\sqrt{2 \pi}} \int_{-\infty}^{x} e^{-t^{2} / 2} d t .
\end{gathered}
$$

Всюду в дальнейшем будем предполагать, что с.в. $T_{1}, \ldots, T_{k_{n}}$ удовлетворяют условию Линдеберга:

$$
L_{n}=\frac{1}{B_{k_{n}}^{2}} \sum_{i=1}^{k_{n}} \int_{\left|X-\mathbf{E} T_{i}\right|>\varepsilon B_{k_{n}}}\left(X-\mathbf{E} T_{i}\right)^{2} d \mathbf{P}\left\{T_{i}<X\right\} \longrightarrow 0 \quad \forall \varepsilon>0 .
$$

Поскольку мы будем иметь дело в основном с одинаково распределенными с.в., то и классификацию предельного поведения распределения $\nu$ проведем именно для этого случая. Предварительно положим

$$
a_{n}=\mathbf{E} T_{1}, \quad \sigma_{n}^{2}=\mathbf{D} T_{1}, \quad \theta_{n}=\left(n a_{n}\right)^{-1}, \quad \widehat{\sigma}_{n}=n \sigma_{n} .
$$

Параметр $\theta_{n}$ играет роль регулирующего, подобно параметру $\rho / n$ в модели (1.2). В связи с этим отметим, что $\theta_{n}=\rho / n$, если $T_{1} \in \exp (\rho)$. По отношению к параметру $\theta_{n}$ будем рассматривать следующие основные случаи: 1) регулярный: $0<\varepsilon<\theta_{n}<1-\varepsilon$ или $1+\varepsilon<\theta_{n}<1 / \varepsilon \quad \forall \varepsilon$; 2) переходный первого типа: $\theta_{n} \rightarrow \infty$; 3) пороговый: $\theta_{n} \rightarrow 1$; 4) переходный второго типа: $\theta_{n} \rightarrow 0$. При этом случай 4) будет рассматриваться при произвольных $m$, случай $0<\varepsilon<\theta_{n}<1-\varepsilon$ при $m=O(n)$, а остальные - при $m=o(n)$. Как будет ясно из дальнейшего, это не ограничивает общности.

При получении интегральных предельных теорем мы будем исходить из следующего представления:

$$
\begin{aligned}
\mathbf{P}\left\{\nu>k_{n}\right\}= & \mathbf{P}\left\{\xi_{1} \leqslant S_{m}, \ldots, \zeta_{k_{n}+1} \leqslant S_{m}\right\} \\
& \times \int \mathbf{P}\left\{\zeta_{1}^{*} \leqslant g_{1}(u), \ldots, \zeta_{k_{n}+1}^{*} \leqslant g_{k_{n}+1}(u)\right\} d \mathbf{P}\left\{S_{m}^{*}<u\right\},
\end{aligned}
$$

где

$$
\begin{gathered}
\zeta_{k}^{*}=\frac{\zeta_{k}-C_{k}}{D_{n}}, \quad S_{m}^{*}=\frac{S_{m}-A_{m}}{B_{m}} \\
g_{k}(u) \equiv g_{n k}(u)=d_{n} u+\frac{A_{m}-C_{k}}{D_{n}}, \quad d_{n}=\frac{B_{m}}{D_{n}} .
\end{gathered}
$$

Далее, если $\zeta_{n}(t)$ - случайная ломаная, построенная по точкам $\left(t_{k}, \zeta_{k}^{*}\right)$, и $g_{n}(t, u)$ - ломаная с вершинами в точках $\left(t_{k}, g_{k}(u)\right)$, $k=0,1, \ldots, k_{n}+1$, то (2.4) может быть записано в виде

$$
\mathbf{P}\left\{\nu>k_{n}\right\}=\int \mathbf{P}\left\{\zeta_{n}(t)<g_{n}(t, u), 0 \leqslant t \leqslant 1\right\} d \mathbf{P}\left\{S_{m}^{*}<u\right\} .
$$


Таким образом, мы свели задачу к принципу инвариантности для сумм независимых с.в., но с подвижной относительно исходных параметров границей. Согласно принципу инвариантности, можно сконструировать $\zeta_{n}(t)$ и стандартный винеровский процесс $w(t)$ на одном вероятностном пространстве так, что (см. [10]) $\zeta_{n}(t)=w(t)+\varepsilon_{n}(t)$, где случайный процесс $\varepsilon_{n}(t)$ обладает тем свойством, что

$$
\varepsilon_{n} \equiv \sup _{0 \leqslant t \leqslant 1}\left|\varepsilon_{n}(t)\right| \stackrel{\mathbf{P}}{\longrightarrow} 0, \quad \text { т.e. } \varepsilon_{n}=o_{p}(1) .
$$

В силу этого из (2.5) имеем

$$
\mathbf{P}\left\{\nu>k_{n}\right\}=\int \mathbf{P}\left\{w(t)<g_{n}(t, u)+o_{p}(1), 0 \leqslant t \leqslant 1\right\} d \mathbf{P}\left\{S_{m}^{*}<u\right\} .
$$

Таким образом, остается изучить асимптотическое поведение границы $g_{n}(t, u)$ при $n \rightarrow \infty$ в зависимости от остальных параметров. Сразу отметим, что в большинстве рассматриваемых случаев предельная граница $g(t, u)$ будет вырожденной.

3. Идеи доказательства и вспомогательные результаты. Одна из теорем (теорема 4.1) в переходном случае первого типа доказывается без использования соотношения (2.6). В условиях этой теоремы задача сводится к изучению момента первого выхода через асимптотически постоянную границу сумм н.о.р.с.в. с показательным распределением.

Доказательство других теорем основывается на представлении (2.6) и подразделяется на два основных случая. В первом из них предельная граница $g(t, \circ)$ является непрерывной функцией (теорема 4.4) и какихлибо проблем с применением принципа инвариантности не возникает. Во втором же она является вырожденной, а именно, $g(t, \circ)=\infty$ при $0<t<1$. При этом возникает необходимость в дополнительном обосновании применения принципа инвариантности в такой ситуации.

Лемма 3.1 ([11]). Если $h_{n}(t)$ - невозрастающая функиия на $\left(\tau_{n}, 1\right], \lim _{n \rightarrow \infty} \tau_{n}=1 u \lim _{n \rightarrow \infty} h_{n}(1)=h_{0}$, mo

$$
\lim _{n \rightarrow \infty} \mathbf{P}\left\{w(t)<h_{n}(t), \tau_{n}<t \leqslant 1\right\}=\Phi\left(h_{0}\right) .
$$

Лемма 3.2. Если $\theta_{n}<1-\varepsilon, \varepsilon>0, m \rightarrow \infty, \beta_{3 n} \equiv \mathbf{E}\left|T_{1}-a_{n}\right|^{3}<\infty$, $\left(\theta_{n} n\right)^{3} \beta_{3 n}=o(m)$, mo $\lim _{n \rightarrow \infty} \mathbf{P}\left\{\nu>n\left(1-\theta_{n}\right)\right\}=1$.

Д оказат ель с т во. Не ограничивая общности, будем считать, что $n\left(1-\theta_{n}\right)$ является целым числом. Тогда из (2.2) и (2.4) имеем

$$
\mathbf{P}\left\{\nu>n\left(1-\theta_{n}\right)-1\right\}=\mathbf{P}\left\{\zeta_{n k}^{0}<A_{m+k}-C_{k}, k=1,2, \ldots, n\left(1-\theta_{n}\right)\right\},
$$

где $\zeta_{n k}^{0}=\zeta_{n k}-\mathbf{E} \zeta_{n k}$. 
Легко видеть, что

$$
\mathbf{P}\left\{\nu>n\left(1-\theta_{n}\right)\right\} \leqslant 1-\sum_{k=1}^{n\left(1-\theta_{n}\right)} \mathbf{P}\left\{\zeta_{n k}^{0} \geqslant A_{m+k}-C_{k}\right\} \equiv 1-\sum_{k=1}^{n\left(1-\theta_{n}\right)} P_{n k} .
$$

В силу неравенства Чебышева

$$
P_{n k} \leqslant \frac{\mathbf{E}\left|\zeta_{n k}^{0}\right|^{3}}{\left(A_{m+k}-C_{k}\right)^{3}} \leqslant \frac{\gamma_{3 k}+(m+k) \beta_{3 n}}{\theta_{n}^{-3} f_{n}^{3}(k / n)},
$$

где

$$
\begin{gathered}
f_{n}(x)=\frac{m}{n}+x+\theta_{n} \ln (1-x) \\
\gamma_{3 k}=\sum_{i=1}^{k} \mathbf{E}\left|\frac{\xi_{i}}{n-i+1}-\frac{1}{n-i+1}\right|^{3} \leqslant \frac{c \cdot k}{n(n-k)^{2}} .
\end{gathered}
$$

Далее, заметим, что $f_{n}(x)$ является вогнутой функцией и поэтому при $0 \leqslant x \leqslant 1-\theta_{n}$ имеем

$$
f_{n}(x) \geqslant \frac{m}{n}+\left(1+\frac{\theta_{n} \ln \theta_{n}}{1-\theta_{n}}\right) x \geqslant \frac{m}{n}+c(\varepsilon) x,
$$

где $c(\varepsilon)=((1-\varepsilon) \ln (1-\varepsilon)) / \varepsilon$.

Теперь из (3.2)-(3.4) имеем

$$
P_{n k} \leqslant C \frac{\theta_{n}^{3}(m+2 k)}{(m+c(\varepsilon) k)^{3}} .
$$

Отсюда при $m \leqslant n\left(1-\theta_{n}\right)$ имеем

$$
\begin{aligned}
\sum_{k=1}^{n\left(1-\theta_{n}\right)} P_{n k}= & \sum_{k=1}^{m} P_{n k}+\sum_{k=m+1}^{n\left(1-\theta_{n}\right)} P_{n k} \leqslant C \theta_{n}^{3} m \sum_{k=1}^{m} \frac{1}{(m+c(\varepsilon) k)^{3}} \\
& +\frac{C \theta_{n}^{3}}{c^{3}(\varepsilon)} \sum_{k=m+1}^{n\left(1-\theta_{n}\right)} \frac{1}{k^{2}} \leqslant C \frac{\theta_{n}^{3}}{\varepsilon^{3} m}+C \frac{\theta_{n}^{3} n^{3} \beta_{3 n}}{c^{3}(\varepsilon)} \sum_{k=m+1}^{\infty} \frac{1}{k^{2}}=o(1) .
\end{aligned}
$$

Утверждение леммы в рассматриваемом случае теперь следует из (3.1). Если $m>n\left(1-\theta_{n}\right)$, то доказательство упрощается.

Далее, приведем некоторые технические результаты, касающиеся показательно распределенных случайных величин.

Лемма 3.3 (см. $\left[9\right.$, гл. 1, §4]). Если $\xi_{1}, \xi_{2}, \ldots$ - независимые одинаково распределенные случайные величины $и \xi_{1} \in \exp (1)$, то

$$
\mathbf{P}\left\{\xi_{1}+\cdots+\xi_{k} \leqslant \lambda, \xi_{1}+\cdots+\xi_{k+1}>\lambda\right\}=\Pi_{k}(\lambda), \quad k=0,1, \ldots
$$

Лемма 3.4. Пусть $\widetilde{S}_{k}$ определены так же, как в соотношежии (2.1). 
1) Ecлu $k_{n} \rightarrow \infty u n-k_{n} \rightarrow \infty$ npu $n \rightarrow \infty$, mo

$$
\mathbf{P}\left\{\frac{\widetilde{S}_{k_{n}}-\mathbf{E} \widetilde{S}_{k_{n}}}{\sqrt{\mathbf{D} \widetilde{S}_{k_{n}}}}<x\right\} \Longrightarrow \Phi(x) .
$$

2) Для любых фиксированных $k=0,1,2, \ldots$ при $n \rightarrow \infty$

$$
\mathbf{P}\left\{\widetilde{S}_{n-k}-\ln n<x\right\} \Longrightarrow G_{k}(x),
$$

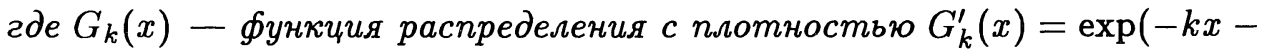
$\left.e^{-x}\right) /(k-1)$ !.

Д ок а з а т е л с с в о. Чтобы доказать утверждение 1), необходимо просто проверить справедливость условия Линдеберга для последовательности $\left\{\tilde{\xi}_{i}, i=1, \ldots, k_{n}\right\}$.

Для доказательства 2) рассмотрим характеристическую функцию

$$
\begin{aligned}
f_{n, k}(t) & =\mathbf{E} \exp \left\{i t\left(\widetilde{S}_{n-k}-\ln n\right)\right\}=e^{-i t \ln n} \prod_{j=k}^{n} \frac{1}{1-i t / j} \\
& \sim e^{-i t\left(\sum_{j=i}^{k-1} 1 / j-c\right)} \prod_{j=k}^{n} \frac{e^{i t / j}}{1-i t / j},
\end{aligned}
$$

где $c$ - постоянная Эйлера. Переходя в (3.5) к пределу при $n \rightarrow \infty$ и учитывая следующее представление для гамма-функции:

$$
\Gamma(z)=\frac{e^{i t z}}{z \prod_{k=1}^{\infty}(1+z / k) e^{-z / k}},
$$

получим $\lim _{n \rightarrow \infty} f_{n, k}(t)=\Gamma(k-i t) / \Gamma(k)$. Соответствующую плотность распределения легко найти с помощью формулы обращения.

Заметим, что подобная лемма для случайных величин с геометрическими распределениями была доказана в работе автора [12].

Перейдем теперь к формулировке и доказательству основных утверждений в соответствии с проведенной выше классификацией предельных случаев.

4. Формулировки основных утверждений.

4.1. Переходный случай первого типа $\left(\theta_{n} \rightarrow \infty\right)$. Первая из приводимых ниже теорем относится к случаю неодинаково распределенных с.в. $T_{1}, T_{2}, \ldots$

Теорема 4.1. Пусть $L_{1}, \ldots, L_{n}$ - независимые одинаково распределенные случайные величины такие, что $L_{1} \in \exp (1), a T_{1}, \ldots$, $T_{m}, \ldots$, - не зависящие от них неотрицательные независимые случайные величины, удовлетворяющие условию (2.3) и такие, что для любых 
фиксированных $k=0,1, \ldots \lim _{n \rightarrow \infty} n A_{m+k}=a<\infty, \lim _{n \rightarrow \infty} n B_{m+k}=$ $b<\infty$. Тогда для любого $k \geqslant 0$

$$
\lim _{n \rightarrow \infty} \mathbf{P}\{\nu=k\}= \begin{cases}\Pi_{k}(a), & b=0, \\ \delta_{0 k} \Phi\left(-\frac{a}{b}\right)+\int_{0}^{\infty} \Pi_{k}(x) d \Phi\left(\frac{x-a}{b}\right), & b>0\end{cases}
$$

где $\delta_{i j}$ - символ Кронекера.

Следствие 4.1.1. Если $T_{1}, T_{2}, \ldots$ - независимые одинаково распределенные случайные величины, $\lim _{n \rightarrow \infty} \theta_{n}=\infty, \lim _{n \rightarrow \infty} m / \theta_{n}=$ $a<\infty, \lim _{n \rightarrow \infty} \sqrt{m} \widehat{\sigma}_{n}=b<\infty$, то (4.1) имеет место.

Всюду в дальнейшем $T_{1}, T_{2}, \ldots$ - н.о.р.с.в., удовлетворяющие условию (2.3), а $L_{1}, \ldots, L_{n}-$ те же, что и в теореме 4.1 .

Теорема 4.2. Если $\lim _{n \rightarrow \infty} \theta_{n}=\infty, \lim _{n \rightarrow \infty} m / \theta_{n}=\infty, m=o(n)$, $\widehat{\sigma}_{n}^{2}=o\left(m / \theta_{n}^{2}\right), m o$

$$
\mathbf{P}\left\{\nu>\frac{m}{\theta_{n}-1}-\sqrt{\frac{m}{\theta_{n}}\left(1+\theta_{n} \widehat{\sigma}_{n}^{2}\right) x}\right\} \Longrightarrow \Phi(x) .
$$

4.2. Регулярный случай $\left(0<\varepsilon<\theta_{n}<1-\varepsilon, 1+\varepsilon<\theta_{n}<1 / \varepsilon\right)$.

Теорема 4.3. Пусть выполнено одно из следующих условий: 1) $0<\varepsilon<\theta_{n}<1-\varepsilon, m=O(n)$ и, кроме того, либо $m=O\left(\sqrt{n\left(1+\widehat{\sigma}_{n}^{2}\right)}\right)$ $u n^{3} \beta_{3 n}=o(m)$, либо $m / \sqrt{n\left(1+\widehat{\sigma}_{n}^{2}\right)} \rightarrow \infty$; 2$) 1+\varepsilon<\theta_{n}<1 / \varepsilon, m=o(n)$, $\widehat{\sigma}_{n}^{2}=o(m)$. Тогда прu $n \rightarrow \infty$

$$
\mathbf{P}\left\{\nu-\gamma_{n}>-b_{n} x\right\} \Longrightarrow \Phi(x),
$$

гдe

$$
\begin{aligned}
& \gamma_{n}= \begin{cases}n \alpha_{n}, & \theta_{n}<1, \\
\frac{m}{\theta_{n}-1}, & \theta_{n}>1,\end{cases} \\
& b_{n}= \begin{cases}\frac{\theta_{n} \sqrt{n\left(1-\alpha_{n}\right)\left[\alpha_{n}+\left(1-\alpha_{n}\right) \widehat{\sigma}_{n}^{2}\left(\alpha_{n}+m / n\right)\right]}}{\alpha_{n}-1+\theta_{n}}, & \theta_{n}<1, \\
\sqrt{m \theta_{n}^{2}\left(1+\theta_{n} \widehat{\sigma}_{n}^{2}\right) /\left(\theta_{n}-1\right)^{3}}, & \theta_{n}>1,\end{cases}
\end{aligned}
$$

а $\alpha_{n}$ - положительный корень уравнения

$$
f_{n}(x) \equiv \frac{m}{n}+x+\theta_{n} \ln (1-x)=0 .
$$

3 а м е ч а н и е. Из условия $n^{3} \beta_{3 n}=o(m)$ в силу неравенства между моментами следует, что $\widehat{\sigma}_{n}^{2}=o\left(m^{2 / 3}\right)$. 
4.3. Пороговый случай $\left(\theta_{n} \rightarrow 1\right)$.

Теорема 4.4. Eсли

$$
\begin{gathered}
\frac{m}{n\left(1+\widehat{\sigma}_{n}^{2}\right)} \longrightarrow \infty, \quad \beta_{n} \equiv \frac{m\left(\theta_{n}-1\right)}{1+\widehat{\sigma}_{n}^{2}} \longrightarrow \beta_{0}, \quad\left|\beta_{0}\right|<\infty, \\
\frac{m^{3}}{n\left(1+\widehat{\sigma}_{n}^{2}\right)} \longrightarrow \gamma_{0}<\infty,
\end{gathered}
$$

mо для любого $x>0$

$$
\mathbf{P}\left\{\nu>\frac{m^{2} x}{1+\widehat{\sigma}_{n}^{2}}\right\} \Longrightarrow \mathbf{P}\left\{w(t)<g_{x}(t), 0 \leqslant t \leqslant 1\right\},
$$

гde

$$
g_{x}(t)=\left[x^{-1 / 2}-\beta_{0} x^{1 / 2} t-\gamma_{0} x^{3 / 2} \frac{t^{2}}{2}\right] .
$$

Следствие 4.4.1. Если в предположениях теоремы $4.4 \gamma_{0}=0$, $\beta_{0} \neq 0$, то для любого $x>0$

$$
\mathbf{P}\left\{\nu>\frac{m^{2} x}{1+\widehat{\sigma}_{n}^{2}}\right\} \Longrightarrow 1-e^{\beta_{0}-\left|\beta_{0}\right|} \int_{0}^{\beta_{0} x q} P_{q^{2}}(u) d u,
$$

где $q=\beta_{0} / 2, P_{\alpha}(u)=\sqrt{\alpha /\left(\pi u^{3}\right)} \exp (2 \sqrt{\alpha}-u-\alpha / u), \alpha>0, u>0$.

Следствие 4.4.2. Если $\beta_{0}=\gamma_{0}=0$, то для любого $x>0$

$$
\mathbf{P}\left\{\nu>\frac{m^{2} x}{1+\widehat{\sigma}_{n}^{2}}\right\} \Longrightarrow 2 \Phi\left(\frac{1}{\sqrt{x}}\right)-1 .
$$

Теорема 4.5. Если $\theta_{n} \rightarrow 1, m\left|1-\theta_{n}\right| \rightarrow \infty, m=o(n), 1-\theta_{n}=$ $o(\sqrt{m / n}), \widehat{\sigma}_{n}^{2}=o\left(\sqrt{m^{3} / n}\right), m o$

$$
\mathbf{P}\left\{\nu-\sqrt{2 m n}>-b_{n} x\right\} \Longrightarrow \Phi(x),
$$

где $b_{n}=\left[n^{3} /(2 m)\right]^{1 / 4}\left(1+\widehat{\sigma}_{n}^{2}\right)^{1 / 2}$.

Теорема 4.6. Если $\theta_{n} \rightarrow 1, m\left|1-\theta_{n}\right| \rightarrow \infty, m=o(n), \sqrt{m / n}=$ $O\left(1-\theta_{n}\right), \widehat{\sigma}_{n}^{2}=o\left(m\left|1-\theta_{n}\right|\right)$ u, кроме того, $\sqrt{m / n} \geqslant \delta\left(1-\theta_{n}\right)$ при $\theta_{n}<1$, $\delta>0, m o$

$$
\mathbf{P}\left\{\nu>\gamma_{n}-b_{n} x\right\} \Longrightarrow \Phi(x)
$$

əde

$$
\begin{aligned}
& \gamma_{n}=\left\{\begin{array}{ll}
n\left(1-\theta_{n}\right)\left(1+\delta_{1}\right), & \theta_{n}<1, \\
\frac{2 m}{\left(\theta_{n}-1\right)\left(1+\delta_{1}\right)}, & \theta_{n}>1,
\end{array} \quad \delta_{1}=\sqrt{1+\frac{2 m}{n\left(1-\theta_{n}\right)^{2}}}\right. \\
& b_{n}= \begin{cases}\left(\frac{n\left(1+\delta_{1}\right)\left(1+\widehat{\sigma}_{n}^{2}\right)}{\left(1-\theta_{n}\right) \delta_{1}^{2}}\right)^{1 / 2}, & \theta_{n}<1, \\
\left(\frac{2 m\left(1+\widehat{\sigma}_{n}^{2}\right)}{\left(\theta_{n}-1\right)^{3}\left(1+\delta_{1}\right) \delta_{1}^{2}}\right)^{1 / 2}, & \theta_{n}>1 .\end{cases}
\end{aligned}
$$


Сразу отметим, что если $T_{1} \in \exp (\rho)$, то из приведенных результатов вытекают все теоремы, полученные А.В. Нагаевым и автором в работе [7].

Далее, как нетрудно заметить, во всех сформулированных теоремах выполняется ограничение $\hat{\sigma}_{n}^{2}=o(m)$. Следующее утверждение показывает, что если $\widehat{\sigma}_{n}^{2}$ и $m$ одного порядка, то картина существенно меняется.

Теорема 4.7. Если $\widehat{\sigma}_{n}^{2} \rightarrow \infty, m \sim c \widehat{\sigma}_{n}^{2}, 0<c<\infty, m=o(n)$, $\theta_{n} \rightarrow \theta_{0}, 0<\theta_{0}<\infty$, то для любого $x>0$

$$
\begin{aligned}
\mathbf{P} & \{\nu>m x\} \\
& \Longrightarrow \int_{0}^{\infty} \mathbf{P}\left\{w(t)<u+\left(\theta_{0}^{-1}-1\right) \sqrt{c x} t, 0 \leqslant t \leqslant 1\right\} d \Phi\left(-\frac{\sqrt{c}}{\theta_{0}}+\sqrt{x} u\right) .
\end{aligned}
$$

Следствие 4.7.1. Если в условиях теоремь $4.7 \theta_{0}=1$, то

$$
\mathbf{P}\{\nu<m x\} \Longrightarrow G(x) \equiv 2 \int_{0}^{\infty} \Phi(\sqrt{x} u-\sqrt{c}) d \Phi(u) .
$$

Легко понять, что распределение $G(x)$ имеет скачок в нуле, так как $G(0)=\Phi(-\sqrt{c})$, т.е. в рассматриваемых условиях имеется положительная вероятность, что величина $\nu$ равна нулю.

4.8. Переходный случай второго типа $\left(\theta_{n} \rightarrow 0\right)$. Пусть $\delta_{n} \equiv$ $\ln n-(m+n) a_{n}=\ln n-(1+m / n) / \theta_{n}, r_{n}=\widehat{\sigma}_{n}^{2} e^{-\delta_{n}} / n$.

Теорема 4.8. Если $m \rightarrow \infty, n \rightarrow \infty, \theta_{n} \equiv\left(n a_{n}\right)^{-1} \longrightarrow 0, \delta_{n} \rightarrow \infty u$ выполнено одно из следующих условий:

1) $m e^{\delta_{n} / 2} /\left(n \theta_{n} \sqrt{1+r_{n}}\right) \longrightarrow \infty$;

2) $m e^{\delta_{n} / 2} /\left(n \theta_{n} \sqrt{1+r_{n}}\right)=O(1),\left(n \theta_{n}\right)^{3} \beta_{3 n}=o(m)$, mo

$$
\mathbf{P}\left\{\nu>n \alpha_{n}-b_{n} x\right\} \Longrightarrow \Phi(x),
$$

где $\alpha_{n}-$ корень уравнения (4.2),

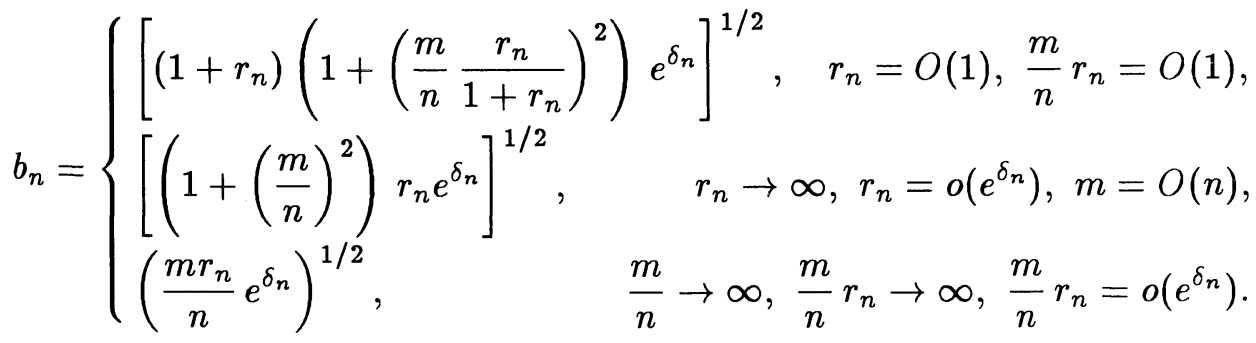

Теорема 4.9. Если $\delta_{n} \rightarrow \delta_{0},\left|\delta_{0}\right|<\infty$, и выполнено одно из следующих условий:

1) $m /\left(\sqrt{n} \theta_{n}\right) \rightarrow \infty, \widehat{\sigma}_{n}=o\left(\min \left(m /\left(\sqrt{n} \theta_{n}\right), n / \sqrt{m+n}\right)\right)$;

2) $m /\left(\sqrt{n} \theta_{n}\right)=O(1),\left(n \theta_{n}\right)^{3} \beta_{3 n}=o(m)$,

то для любого челого фиксированного $k \geqslant 0$

$$
\lim _{n \rightarrow \infty} \mathbf{P}\{n-\nu=k\}=\Pi_{k}\left(e^{\delta_{0}}\right) .
$$


Для специальной схемы серий, образованной с помощью масштабного параметра, теорема 4.9 была получена другим методом Боллом [5] (1986 г.). В случае $T_{1} \in \exp (\rho)$ она была доказана Дэниелсом [13] (1967 г.) для «малых $m$ », а также автором [12] (1971 г.) и Селлке [6] (1983 г.) при произвольных $m$.

\section{5. Доказательства теорем.}

5.1. Доказательство теоремы 4.1. В силу (2.1), (2.2) и (2.4) имеем

$$
\begin{gathered}
\mathbf{P}\{\nu=k\}=\mathbf{P}\left\{\xi_{1} \leqslant n B_{m} S_{m}^{*}+n A_{m}, \ldots, \xi_{1}+\cdots+\xi_{k+1}>n B_{m} S_{m}^{*}\right. \\
+n \sqrt{B_{m+k}^{2}-B_{m}^{2}}\left(S_{m+k}-S_{m}\right)^{*}+n A_{m+k} \\
\left.-\left(\frac{\xi_{2}}{n-1}+\cdots+\frac{k}{n-k} \xi_{k+1}\right)\right\} \\
=\int \mathbf{P}\left\{\begin{array}{l}
\xi_{1} \leqslant a+b x+o_{p}(1), \ldots, \xi_{1}+\cdots \\
\left.\cdots+\xi_{k+1}>a+b x+o_{p}(1)\right\} d \mathbf{P}\left\{S_{m}^{*}<x\right\},
\end{array}\right.
\end{gathered}
$$

где $o_{p}(1) \stackrel{\mathbf{P}}{\longrightarrow} 0$, а $\xi_{1}, \xi_{2}, \ldots-$ н.о.р.с.в. такие, что $\xi_{1} \in \exp (1)$.

Отсюда в силу центральной предельной теоремы и леммы 3.2 получим для любого $k \geqslant 1$

$$
\lim _{n \rightarrow \infty} \mathbf{P}\{\nu=k\}=\int_{-a / b}^{\infty} \Pi_{k}(a+b x) d \Phi(x)=\int_{0}^{\infty} \Pi_{k}(x) d \Phi\left(\frac{x-a}{b}\right) .
$$

Для $k=0$ имеем

$$
\begin{aligned}
\lim _{n \rightarrow \infty} \mathbf{P}\{\nu=0\} & =\int_{-\infty}^{\infty} \mathbf{P}\left(\xi_{1}>a+b x\right) d \Phi(x) \\
& =\Phi\left(-\frac{a}{b}\right)+\int_{-a / b}^{\infty} \Pi_{0}(a+b x) d \Phi(x),
\end{aligned}
$$

что и завершает доказательство.

5.2. Доказательство теорем 4.4 и 4.7. Начнем с теоремы 4.4. В соответствии с (2.6) и (4.3) нам необходимо показать, что

$$
\lim _{n \rightarrow \infty} g_{n}(t, u)=g_{x}(t) \equiv \frac{1}{\sqrt{x}}-\beta_{0} \sqrt{x} t-\gamma_{0} \sqrt{x^{3}} \frac{t^{2}}{2} .
$$

Из $(2.2),(2.4)$ и (2.5) имеем

$$
g_{n}\left(t_{k}, u\right) \equiv g_{k}(u)=d_{n} u+\frac{A_{m}-C_{k}}{D_{n}} .
$$


Таким образом, необходимо изучить асимптотическое поведение $g_{k}(u)$ при $k \leqslant k_{n}=\left[m^{2} x /\left(1+\widehat{\sigma}_{n}^{2}\right)\right]$. Не ограничивая общности, будем считать, что $m^{2} x /\left(1+\widehat{\sigma}_{n}^{2}\right)$ является целым числом.

Поскольку $k_{n}=\cdot o(n)$, имеем при $k \rightarrow \infty, k \leqslant k_{n}, m \rightarrow \infty$ и $n \rightarrow \infty$ следующие асимптотические соотношения:

$$
\begin{aligned}
D_{n}^{2} & =\mathbf{D} \zeta_{k_{n}+1}=\mathbf{D}\left(\frac{\xi_{1}}{n}+\cdots+\frac{\xi_{k_{n}+1}}{n-k_{n}}-T_{m+1}-\cdots-T_{m+k_{n}}\right) \\
& =k_{n} \sigma_{n}^{2}+\frac{k_{n}}{n\left(n-k_{n}\right)}+O\left(\frac{1}{n^{2}}\right), \\
C_{k} & =\mathbf{E} \zeta_{k+1}=\frac{1}{n-k}+\cdots+\frac{1}{k}-k a_{n} \\
& =-\ln \left(1-\frac{k}{n}\right)-k a_{n}+O\left(\frac{1}{n-k}\right), \\
t_{k} & =\frac{D_{k}^{2}}{D_{n}^{2}}=\frac{k_{n} \sigma_{n}^{2}+k /(n(n-k))+O\left(1 / n^{2}\right)}{k_{n} \sigma_{n}^{2}+k_{n} /\left(n\left(n-k_{n}\right)\right)+O\left(1 / n^{2}\right)} \\
& =\frac{k}{k_{n}} \frac{1+\widehat{\sigma}_{n}^{2}+o(1)}{1+\widehat{\sigma}_{n}^{2}+o(1)} \sim \frac{k}{k_{n}}, \\
d_{n} & =\frac{B_{m}^{2}}{D_{n}^{2}} \sim \frac{m \widehat{\sigma}_{n}^{2}}{k_{n}\left(1+\widehat{\sigma}_{n}^{2}\right)}=\frac{\widehat{\sigma}_{n}^{2}}{m x} .
\end{aligned}
$$

Отсюда следует, что

$$
\begin{aligned}
g_{k}(u) \sim & \frac{m a_{n}+k a_{n}+b_{n}(1-k / n)}{\sqrt{k_{n} \sigma_{n}^{2}+k_{n} /\left(n\left(n-k_{n}\right)\right)}} \\
\sim & \frac{m+\left(1-\theta_{n}\right) k-\left(\theta_{n} / 2\right) k^{2} / n+o\left(k^{2} / n^{2}\right)}{\sqrt{k_{n}\left(1+\widehat{\sigma}_{n}^{2}\right)}} \\
= & \frac{m}{\sqrt{k_{n}\left(1+\widehat{\sigma}_{n}^{2}\right)}}-\frac{\sqrt{k_{n}}\left(\theta_{n}-1\right)}{\sqrt{1+\widehat{\sigma}_{n}^{2}}} \frac{k}{k_{n}} \\
& -\frac{\theta_{n} k_{n}^{3 / 2}}{2 n \sqrt{1+\widehat{\sigma}_{n}^{2}}}\left(\frac{k}{k_{n}}\right)^{2}+o(1) \sim g_{x}(t),
\end{aligned}
$$

если $k \sim t k_{n}, 0<t<1$, что и требовалось доказать.

Что касается следствия 4.4.1, то его утверждение опирается на явную формулу для вероятности $\mathbf{P}\{w(t)<a+b t, 0 \leqslant t \leqslant 1\}$, в нужном нам виде эту формулу можно найти в [10].

В условиях теоремы 4.7 имеем $k_{n}=m^{2} x / \widehat{\sigma}_{n}^{2}, d_{n}^{2} \sim \widehat{\sigma}_{n}^{2} /(m x) \sim 1 /(c x)$, $g_{k}(u) \sim u / \sqrt{c x}+\left(1 / \theta_{0}\right)\left(1 / \sqrt{x}+\sqrt{x}\left(1-\theta_{0}\right) c \theta_{k}\right)$ и в силу этого

$$
\begin{aligned}
\mathbf{P}\left\{\nu>\frac{m^{2} x}{\widehat{\sigma}_{n}^{2}}\right\} \sim \int_{u>-\sqrt{c} / \theta_{0}} \mathbf{P}\{ & w(t)<\frac{u}{\sqrt{c x}}+\frac{1}{\theta_{0} \sqrt{x}} \\
& \left.+\sqrt{x}\left(\frac{1}{\theta_{0}}-1\right) c t, 0 \leqslant t \leqslant 1\right\} d \Phi(x),
\end{aligned}
$$

откуда легко следует утверждение теоремы. 
5.3. Доказательства теорем 4.2, 4.3, 4.5, 4.6 и 4.8. Общей чертой доказательства этих теорем является то, что предельная граница $g(t, u)$ вырождена в указанном ранее смысле, следствием чего и является возникновение нормального закона в качестве предельного.

Д ок аз а т е ль с т в о т е о р е мы 4.3. Пусть сначала $0<\varepsilon<$ $\theta_{n}<1-\varepsilon$ и $m / \sqrt{n\left(1+\widehat{\sigma}_{n}^{2}\right)} \rightarrow \infty$. В этом случае возьмем $k_{n}=n \alpha_{n}-b_{n}^{\prime} x$, где $\alpha_{n}-$ корень уравнения $f_{n}(x)=0$ (см. (4.2)), а последовательность $b_{n}^{\prime}=o(n)$ будет выбрана позже.

Исходя из (5.1) с учетом (5.2) имеем

$$
\begin{aligned}
d_{n}^{2} & \sim \frac{m}{n \alpha_{n}} \frac{\widehat{\sigma}_{n}^{2}}{\widehat{\sigma}_{n}^{2}+\left(1-\alpha_{n}\right)^{-1}}=O(1), \\
g_{n}\left(t_{k}, u\right) & \sim d_{n} u+\frac{m a_{n}+k a_{n}+\ln (1-k / n)}{\sqrt{n \alpha_{n} \sigma_{n}^{2}+\alpha_{n} /\left(n\left(1-\alpha_{n}\right)\right)}}=d_{n} u+b_{n}^{\prime \prime} f_{n}\left(\frac{k}{n}\right),
\end{aligned}
$$

где

$$
b_{n}^{\prime \prime}=\frac{1}{\theta_{n}}\left(\frac{n\left(1-\alpha_{n}\right)}{\alpha_{n}\left(1+\left(1-\alpha_{n}\right) \hat{\sigma}_{n}^{2}\right)}\right)^{1 / 2} .
$$

Отсюда легко находим, что

$$
\begin{aligned}
g_{n}(1, u) & \sim b_{n}^{\prime \prime} f_{n}\left(\frac{k_{n}}{n}\right)+d_{n} u=d_{n} u+b_{n}^{\prime \prime}\left[f_{n}^{\prime}\left(\alpha_{n}\right)\left(\frac{k_{n}}{n}-\alpha_{n}\right)+o\left(\frac{k_{n}}{n}-\alpha\right)\right] \\
& \sim d_{n} u-\frac{b_{n}^{\prime \prime}}{n} f_{n}^{\prime}\left(\alpha_{n}\right) b_{n}^{\prime} x=d_{n} u+x
\end{aligned}
$$

если

$$
b_{n}^{\prime}=-\frac{n}{b_{n}^{\prime \prime} f_{n}^{\prime}\left(\alpha_{n}\right)}=\frac{\theta_{n}}{\alpha_{n}-1+\theta_{n}}\left[n \alpha_{n}\left(1-\alpha_{n}\right)\left(1+\left(1-\alpha_{n}\right) \widehat{\sigma}_{n}^{2}\right)\right]^{1 / 2} .
$$

Из (5.3) следует, что если $k_{n}^{\prime}=n \alpha_{n}-b_{n}^{\prime} x_{1}$, где $x_{1} \rightarrow \infty, x_{1}=o\left(n \alpha_{n} / b_{n}^{\prime}\right)$, то $t_{k_{n}^{\prime}} \rightarrow 1$ и при этом $g_{n}\left(t_{k_{n}^{\prime}}, u\right) \sim x_{1} \rightarrow \infty$.

Заметим, что в рассматриваемом случае $g_{n}(0, u) \rightarrow \infty$. Отсюда следует в силу вогнутости $f_{n}(x)$, что $\mathbf{P}\left\{\nu>k_{n}^{\prime}\right\} \rightarrow 1$. Теперь из (2.6) и леммы 3.1 легко получаем

$$
\begin{aligned}
\mathbf{P}\left\{\nu>n \alpha_{n}-b_{n}^{\prime} x\right\} & =\int \Phi\left(d_{n} u+x\right) d \Phi(u)+o(1) \\
& =\Phi\left(\frac{x}{\sqrt{1+d_{n}^{2}}}\right)+o(1) .
\end{aligned}
$$

Если выбрать $b_{n}=\sqrt{1+d_{n}^{2}} b_{n}^{\prime}$, то отсюда следует утверждение теоремы в рассматриваемом случае.

Если же $m=O\left(\sqrt{n\left(1+\widehat{\sigma}_{n}^{2}\right)}\right)$, то нужный результат получается с использованием леммы 3.2 .

Пусть теперь $1+\varepsilon<\theta_{n}<1 / \varepsilon, m \rightarrow \infty, m=o(n)$. В этом случае уравнение $f_{n}(x) \equiv m / n+x+\theta_{n} \ln (1-x)=0$ имеет асимптотическое 
решение $\alpha_{n}=m /\left(n\left(\theta_{n}-1\right)\right)$. Полагая $k_{n}=n \alpha_{n}-b_{n}^{\prime} x=m /\left(\theta_{n}-1\right)-b_{n}^{\prime} x$, где $b_{n}^{\prime}=o\left(m /\left(\theta_{n}-1\right)\right)$, в силу (5.2) имеем

$d_{n}^{2} \sim \frac{\left(\theta_{n}-1\right) \hat{\sigma}_{n}^{2}}{1+\widehat{\sigma}_{n}^{2}}=O(1)$,
$g_{n}\left(t_{k_{1}}, u\right) \sim d_{n} u+\frac{m a_{n}+k a_{n}+\ln (1-k / n)}{\sqrt{\left(m /\left(\theta_{n}-1\right)\right) \sigma_{n}^{2}+m /\left(n^{2}\left(\theta_{n}-1\right)\right)}} \sim d_{n} u+b_{n}^{\prime \prime} f_{n}\left(\frac{k}{n}\right)$,

где $b_{n}^{\prime \prime}=\left(n / \theta_{n}\right) \sqrt{\left(\theta_{n}-1\right) /\left(m\left(1+\widehat{\sigma}_{n}^{2}\right)\right)}$. По аналогии с (5.3) получим, что $g_{n}(1, u) \sim d_{n} u+x$, если $b_{n}^{\prime}=-n /\left(f_{n}^{\prime}\left(\alpha_{n}\right) b_{n}^{\prime \prime}\right)$, т.е. $b_{n}^{\prime} \sim\left[\theta_{n}^{2} m\left(1+\widehat{\sigma}_{n}^{2}\right) /\right.$ $\left.\left(\theta_{n}-1\right)^{3}\right]^{1 / 2}$.

Заметим,что в данном случае

$$
g_{n}(0, u) \sim b_{n}^{\prime \prime} f_{n}(0) \sim\left(\frac{\theta_{n}-1}{\theta_{n}} \frac{m}{1+\widehat{\sigma}_{n}^{2}}\right)^{1 / 2} \longrightarrow \infty
$$

Теперь, так же, как и при получении (5.4), имеем

$$
\mathbf{P}\left\{\nu_{n}>n \alpha_{n}-b_{n} x\right\} \Longrightarrow \Phi(x),
$$

где

$$
b_{n}=\sqrt{1+d_{n}^{2}} b_{n}^{\prime}=\left(\frac{m \theta_{n}^{2}\left(1+\theta_{n}^{2} \widehat{\sigma}_{n}^{2}\right)}{\left(\theta_{n}-1\right)^{3}}\right)^{1 / 2} .
$$

Тем самым теорема 4.3 доказана полностью.

Д оказат ельст во т е о р е ы 4.2. Если $\widehat{\sigma}_{n}=o(1), \theta_{n} \widehat{\sigma}_{n}^{2}=$ $O(1)$, то доказательство второго случая предыдущей теоремы проходит практически без изменений и при этом

$$
d_{n} \sim \theta_{n} \widehat{\sigma}_{n}^{2}, \quad g_{n}(0, u) \sim \sqrt{\frac{m}{\theta_{n}}} \rightarrow \infty, \quad b_{n}^{2} \sim \frac{m\left(1+\theta_{n} \widehat{\sigma}_{n}^{2}\right)}{\theta_{n}} .
$$

Если же $\theta_{n} \widehat{\sigma}_{n}^{2} \rightarrow \infty$, то $d_{n} \rightarrow \infty$, и тогда, исходя из $(2.4)$, имеем

$$
\begin{aligned}
\mathbf{P}\left\{\nu>k_{n}\right\} & =\mathbf{P}\left\{\zeta_{k} \leqslant S_{m}, 1 \leqslant k \leqslant k_{n}+1\right\} \\
& =\mathbf{P}\left\{\zeta_{k}^{*} d_{n}^{-1}-\frac{A_{m}-C_{k}}{B_{m}} \leqslant S_{m}^{*}, 1 \leqslant k \leqslant k_{n}+1\right\} \\
& =\mathbf{P}\left\{S_{m}^{*} \geqslant-\tilde{g}_{n, k}+o_{p}(1), 1 \leqslant k \leqslant k_{n}+1\right\} .
\end{aligned}
$$

Теперь, используя (5.2), находим

$$
\tilde{g}_{n, k} \equiv \frac{A_{m}-C_{k}}{B_{m}} \sim \frac{m a_{n}+k a_{n}+\ln (1-k / n)}{\sqrt{m \sigma_{n}^{2}}} \sim \frac{n f_{n}(k / n)}{\sqrt{m \theta_{n}^{2} \widehat{\sigma}_{n}^{2}}} .
$$

А так как $f_{n}(x)$ убывает на $[0,1)$ при $\theta_{n}>1$, из $(5.3)$ получим

$$
\mathbf{P}\left\{\nu>k_{n}\right\} \sim \mathbf{P}\left\{S_{m}^{*} \geqslant-\widetilde{g}_{n, k_{n}}\right\} \sim 1-\Phi(-x)=\Phi(x),
$$


если $k_{n}=m /\left(\theta_{n}-1\right)-b_{n} x$, где $b_{n}=\sqrt{m \widehat{\sigma}_{n}^{2}}=\sqrt{\left(m / \theta_{n}\right) \theta_{n} \widehat{\sigma}_{n}^{2}}$ и при этом $b_{n}=o\left(m / \theta_{n}\right)$ в силу условия $\theta_{n} \widehat{\sigma}_{n}^{2}=o\left(m / \theta_{n}\right)$. Теорема доказана.

Док аз а т ельст то т еоремы 4.5. В условиях теоремы имеем следующее асимптотическое поведение корня уравнения $f_{n}(x)=0$ : $\alpha_{n} \sim \sqrt{2 m / n}$. Полагая, как и ранее, $k_{n}=n \alpha_{n}-b_{n} x$, получим

$$
d_{n}^{2} \sim \sqrt{\frac{m}{2 n}} \frac{\widehat{\sigma}_{n}^{2}}{1+\widehat{\sigma}_{n}^{2}}=o(1),
$$

$g_{k_{n}}(u) \sim \frac{m a_{n}+k a_{n}+\ln \left(1-k_{n} / n\right)}{\sqrt{k_{n} \sigma_{n}^{2}+k_{n} /\left(n\left(n-k_{n}\right)\right)}} \sim \frac{n^{5 / 4}}{\sqrt[4]{2 m}} f_{n}\left(\frac{k_{n}}{n}\right)\left(1+\widehat{\sigma}_{n}^{2}\right)^{-1 / 2}=x$

если

$$
b_{n}=-\frac{\sqrt[4]{2 m n\left(1+\widehat{\sigma}_{n}^{2}\right)}}{f_{n}^{\prime}\left(\alpha_{n}\right)} \sim\left(\frac{n^{3}}{2 m}\right)^{1 / 4} \sqrt{1+\widehat{\sigma}_{n}^{2}} .
$$

Далее, ввиду условия $\widehat{\sigma}_{n}^{2}=o\left(\sqrt{m^{3} / n}\right)$ имеем

$$
b_{n}=o\left(n \alpha_{n}\right), \quad g_{n}(0, u) \sim\left(\frac{m^{3}}{2 n}\right)^{1 / 4}\left(1+\widehat{\sigma}_{n}^{2}\right)^{-1 / 2} \longrightarrow \infty .
$$

Д ок аз а т ель с т в о т е о ре мы 4.6. Схема доказательства остается прежней. В условиях данной теоремы имеем

$$
\begin{aligned}
& d_{n} \sim\left\{\begin{array}{ll}
\frac{2 m}{n\left(\theta_{n}-1\right)\left(1+\delta_{1}\right)}, & \theta_{n}>1, \\
\left(1-\theta_{n}\right)\left(1+\delta_{1}\right), & \theta_{n}<1,
\end{array}, \quad \delta_{1}=\sqrt{1+\frac{2 m}{n\left(1-\theta_{n}\right)^{2}}},\right. \\
& d_{n}^{2} \sim \begin{cases}\frac{\left(\theta_{n}-1\right)\left(1+\delta_{1}\right) \hat{\sigma}_{n}^{2}}{2\left(1+\widehat{\sigma}_{n}^{2}\right)}=o(1), & \theta_{n} \downarrow 1, \\
\frac{m}{n\left(1-\theta_{n}\right)} \frac{\widehat{\sigma}_{n}^{2}}{\left(1+\delta_{1}\right)\left(1+\widehat{\sigma}_{n}^{2}\right)}=o(1), & \theta_{n} \uparrow 1,\end{cases} \\
& b_{n}^{2} \sim \begin{cases}\frac{n\left(1+\delta_{1}\right)\left(1+\hat{\sigma}_{n}^{2}\right)}{\left(1-\theta_{n}\right) \delta_{1}^{2}}, & \theta_{n}<1, \\
\frac{2 m\left(1+\widehat{\sigma}_{n}^{2}\right)}{\left(\theta_{n}-1\right)^{3}\left(1+\delta_{1}\right) \delta_{1}^{2}}, & \theta_{n}>1,\end{cases} \\
& g_{n}(0, u) \sim \frac{m}{\sqrt{n \alpha\left(1+\widehat{\sigma}_{n}^{2}\right)}} \sim \begin{cases}\sqrt{\frac{\left(\theta_{n}-1\right) m\left(1+\delta_{1}\right)}{2\left(1+\widehat{\sigma}_{n}^{2}\right)},} & \theta_{n}>1, \\
\sqrt{\frac{m^{2}}{n\left(1-\theta_{n}\right)\left(1+\widehat{\sigma}_{n}^{2}\right)\left(1+\delta_{1}\right)}}, & \theta_{n}<1 .\end{cases}
\end{aligned}
$$

Легко видеть, что в условиях теоремы $g_{n}(0, u) \rightarrow \infty$ и условие $b_{n}=o\left(n \alpha_{n}\right)$ также имеет место.

Д ок аз а т ель с т в о т е о ремы 4.8. Если $\alpha_{n}$ - положительный корень уравнения $f_{n}(x)=m / n+x+\theta_{n} \ln (1-x)=0$, то мы имеем в предположениях теоремы $\alpha_{n} \rightarrow 1, n\left(1-\alpha_{n}\right) \sim e^{\delta_{n}} \rightarrow \infty$. 
Таким образом, если $k_{n}=n \alpha_{n}-b_{n}^{\prime} x$, где $b_{n}^{\prime}=o\left(n\left(1-\alpha_{n}\right)\right)$, то

$$
\begin{aligned}
D_{n}^{2} & =k_{n} \sigma_{n}^{2}+\frac{k_{n}}{n\left(n-k_{n}\right)}+O\left(\frac{1}{\left(n-k_{n}\right)^{2}}\right) \\
& \sim \frac{1+\left(1-\alpha_{n}\right) \hat{\sigma}_{n}^{2}}{n\left(1-\alpha_{n}\right)}=\left(1+r_{n}\right) e^{-\delta_{n}} \\
d_{n}^{2} & =\frac{m \sigma_{n}^{2}}{D_{n}} \sim \frac{m}{n} \frac{\left(1-\alpha_{n}\right) \hat{\sigma}_{n}^{2}}{1+\left(1-\alpha_{n}\right) \hat{\sigma}_{n}^{2}} \sim \frac{m}{n} \frac{r_{n}}{1+r_{n}} \\
g_{n}(1, u) & \sim d_{n} u+\frac{n a_{n}}{D_{n}} f_{n}\left(\frac{k_{n}}{n}\right) \sim d_{n} u-\frac{n a_{n}}{D_{n}} f_{n}^{\prime}\left(\alpha_{n}\right) \frac{b_{n}^{\prime}}{n} x .
\end{aligned}
$$

Таким образом, $g_{n}(1, u) \sim d_{n} u+x$, если

$$
b_{n}^{\prime}=-\frac{D_{n}}{a_{n} f_{n}^{\prime}\left(\alpha_{n}\right)} \sim n\left(1-\alpha_{n}\right) D_{n} \sim \sqrt{1+r_{n}} e^{\delta_{n} / 2} .
$$

Теперь, выбирая $b_{n}=\sqrt{1+d_{n}^{2}} b_{n}^{\prime}$, как и в (5.4), получим утверждение теоремы в первых двух случаях, участвующих в описании $b_{n}$. При этом ограничение $m e^{\delta_{n} / 2} /\left(n \theta_{n} \sqrt{1+r_{n}}\right) \rightarrow \infty$ обеспечивает выполнение условия $g_{n}(0, u) \rightarrow \infty$. Если же это ограничение не выполнено и при этом $\beta_{3 n}=o\left(m /\left(\theta_{n}^{3} n^{3}\right)\right)$, то необходимо предварительно воспользоваться леммой 3.2 .

В третьем случае величина $d_{n} \rightarrow \infty$ и поэтому, как и в $(5.5)$, имеем

$$
\mathbf{P}\left\{\nu>k_{n}\right\}=\mathbf{P}\left\{S_{m}^{*} \geqslant-\tilde{g}_{n, k}+o_{p}(1), 1 \leqslant k \leqslant k_{n}+1\right\},
$$

где

$$
\widetilde{g}_{n, k}=\frac{A_{m}-C_{k}}{B_{m}} \sim-\frac{n f_{n}(k / n)}{\sqrt{m \theta_{n} \widehat{\sigma}_{n}^{2}}}
$$

Нетрудно проверить, что в рассматриваемом случае $\tilde{g}_{n, 1} \sim$ $\sqrt{m} n a_{n} / \widehat{\sigma}_{n}=\sqrt{m / n} n a_{n} /\left(\widehat{\sigma}_{n} / \sqrt{n}\right) \longrightarrow \infty$ и поэтому в силу вогнутости функции $f_{n}(x)$

$$
\mathbf{P}\left\{\nu>k_{n}\right\} \sim \mathbf{P}\left\{S_{m}^{*} \geqslant-\widetilde{g}_{n, k}\right\} \sim \Phi(x),
$$

если $k_{n}=n \alpha_{n}-b_{n} x$, где $b_{n}=\left(\left(m r_{n} / n\right) e^{\delta_{n}}\right)^{1 / 2}$.

5.4. Доказательство теоремы 4.9. Сначала покажем, что в условиях теоремы $\mathbf{P}\left\{\nu>k_{n}\right\} \rightarrow 1$, если $k_{n}=[n \delta], 0<\delta<1$. Если выполнено условие 2), то это следует из леммы 3.2. Если же выполнено 1$)$, то в соответствии с $(2.4)$ необходимо показать, что $A_{m} / D_{n} \rightarrow \infty$ и $\left(A_{m}-C_{k_{n}}\right) / D_{n} \rightarrow \infty$. Действительно,

$$
\frac{A_{m}}{D_{n}} \sim \frac{m a_{n}}{\sqrt{k_{n} \sigma_{n}^{2}+k_{n} /\left(n\left(n-k_{n}\right)\right)}}=\frac{m}{\theta_{n} \sqrt{n \delta} \sqrt{\hat{\sigma}_{n}^{2}+1 /(1-\delta)}} \rightarrow \infty,
$$

а второе выражение стремится к бесконечности в силу возрастания границы при $k<n\left(1-\theta_{n}\right)$. 
Теперь, опираясь на этот факт, покажем, что $\mathbf{P}\left\{\nu>\ell_{n}\right\}$ также стремится к единице, если $\ell_{n} \rightarrow \infty$ и $h_{n} \equiv n-\ell_{n} \rightarrow \infty$. Для этого достаточно показать, что

$$
\frac{A_{m}-C_{k_{n}}}{\mathbf{D} \zeta_{\ell_{n}+1}} \rightarrow \infty, \quad \frac{A_{m}-C_{\ell_{n}}}{\mathbf{D} \zeta_{\ell_{n}+1}} \rightarrow \infty
$$

Очевидно, что в случае 1 )

$$
\frac{A_{m}-C_{k_{n}}}{\mathbf{D} \zeta_{\ell_{n}+1}} \sim \frac{m / n+\delta+\theta_{n} \ln (1-\delta)}{\theta_{n} \sqrt{\hat{\sigma}_{n}^{2} / n+1 / h_{n}}} \rightarrow \infty .
$$

В случае 2) из условия $\theta_{n}^{3} n^{3} \beta_{3 n}=o(m)$ следует, что $\hat{\sigma}_{n}^{2}=o\left(m^{2 / 3} / \theta_{n}^{2}\right)$, откуда и вытекает требуемое утверждение.

Рассмотрим второе выражение:

$$
\begin{aligned}
\frac{A_{m}-C_{\ell_{n}}}{\mathbf{D} \zeta_{\ell_{n}+1}} & \sim \frac{m a_{n}+a_{n} \ell_{n}+\ln \left(1-\ell_{n} / n\right)}{\sqrt{\hat{\sigma}_{n}^{2} / n+1 / h_{n}}} \\
& =\frac{(m+n) a_{n}-\ln n-h_{n} a_{n}+\ln h_{n}}{\sqrt{\hat{\sigma}_{n}^{2} / n+1 / h_{n}}} \\
& =\frac{\ln h_{n}-h_{n} /\left(n \theta_{n}\right)-\delta_{n}}{\sqrt{\hat{\sigma}_{n}^{2} / n+1 / h_{n}}} \longrightarrow \infty,
\end{aligned}
$$

если $h_{n}=O\left(n \theta_{n}\right)$.

Отсюда следует, что

$$
\mathbf{P}\{\nu>n-k-1\}=\mathbf{P}\left(\Delta_{n}\right)+o(1),
$$

где

$$
\Delta_{n}=\bigcap_{i=\ell_{n}}^{n-k} C_{i}, \quad C_{i}=\left\{\widetilde{S}_{i} \leqslant S_{m+i-1}\right\}
$$

Теперь покажем, что $\mathbf{P}\left(\Delta_{n}\right)=\mathbf{P}\left(C_{n-k}\right)+o(1)$. Заметив, что $\mathbf{P}\left(\Delta_{n}\right)=\mathbf{P}\left(C_{n-k}\right)-\mathbf{P}\left(\cup_{i=\ell_{n}}^{n-k-1} C_{n-k} \bar{C}_{i}\right)$, оценим величину

$$
Q=\mathbf{P}\left(\bigcup_{i=\ell_{n}}^{n-k-1} C_{n-k} \bar{C}_{i}\right) \leqslant \sum_{i=\ell_{n}}^{n-k-1} \mathbf{P}\left(C_{n-k} \bar{C}_{i}\right) \equiv \sum_{i=\ell_{n}}^{n-k-1} Q_{n i} .
$$

Имеем

$$
\begin{aligned}
Q_{n i}= & \mathbf{P}\left\{\widetilde{S}_{n-k} \leqslant S_{m+n-k-1}, \widetilde{S}_{i}>S_{m+i-1}\right\} \\
= & \mathbf{P}\left\{\widetilde{S}_{n-k}-\widetilde{S}_{i} \leqslant S_{m+n-k-1}-S_{m+i-1}+S_{m+i-1}-\widetilde{S}_{i},\right. \\
& \left.\quad S_{m+i-1}-\widetilde{S}_{i}<0\right\} \\
\leqslant & \mathbf{P}\left\{\widetilde{S}_{n-k}-\widetilde{S}_{i} \leqslant T_{m+i}+\cdots+T_{m+n-k-1}\right\} \leqslant \mathbf{P}\left\{\tilde{\xi}_{n-k} \leqslant \widetilde{S}_{n k}\right\},
\end{aligned}
$$

где $\widehat{S}_{n k}=T_{m+\ell_{n}}+\cdots+T_{m+n-k-1}$. 
Заметив, что

$$
\mathbf{E} \widehat{S}_{n k}=\left(h_{n}-k-1\right) a_{n}, \quad \mathbf{D} \widehat{S}_{n k}=\left(h_{n}-k-1\right) \sigma_{n}^{2},
$$

находим

$$
\begin{aligned}
Q_{n i} & \leqslant \mathbf{P}\left\{\tilde{\xi}_{n-k} \leqslant \widehat{S}_{n k}^{*} \sqrt{h_{n}} \sigma_{n}+a_{n} h_{n}+o(1)\right\} \\
& =\int_{-a_{n} \sqrt{h_{n}} / \sigma_{n}}^{+\infty}\left[1-e^{-\left(a_{n} h_{n}+\sigma_{n} \sqrt{h_{n}} x\right)(k+1)}\right] d \Phi(x)+o(1) \\
& \leqslant(k+1) \int_{-\infty}^{+\infty}\left(a_{n} h_{n}+\sigma_{n} \sqrt{h_{n}}|x|\right) d \Phi(x)+o(1) \\
& =(k+1)\left[a_{n} h_{n}+\sigma_{n} \sqrt{h_{n}}\right]+o(1)
\end{aligned}
$$

Теперь, с учетом того, что $a_{n}=\left(\ln n-\delta_{n}\right) /(m+n)$, из $(5.8)$ находим

$$
Q_{n} \leqslant(k+1)\left(h_{n}-k-1\right)\left(a_{n} h_{n}+\frac{\hat{\sigma}_{n} \sqrt{h_{n}}}{n}\right)+o(1)=o(1),
$$

если $h_{n}=o\left(n^{1 / 3}\right)$.

Таким образом, из (5.6)-(5.8) имеем

$$
\begin{aligned}
& \mathbf{P}\{\nu>n-k-1\}=\mathbf{P}\left(C_{n-k}\right)+o(1)=\mathbf{P}\left\{\widetilde{S}_{n-k} \leqslant S_{n+m-k-1}\right\}+o(1) \\
& \quad=\mathbf{P}\left\{\widetilde{S}_{n-k}^{\circ} \leqslant S_{n+m-k-1}^{\circ}+(n+m) a_{n}-\ln n+o(1)\right\}+o(1),
\end{aligned}
$$

где

$$
\widetilde{S}_{n-k}^{\circ}=\widetilde{S}_{n-k}-\ln n, \quad S_{n+m-k-1}^{\circ}=S_{n+m-k-1}-(m+n) a_{n} .
$$

A так как

$$
\mathbf{D} S_{n+m-k-1}^{\circ}=(m+n-k-1) \sigma_{n}^{2} \leqslant \frac{m+n}{n^{2}} \tilde{\sigma}_{n}^{2}=o(1)
$$

то в силу леммы 3.4

$$
\begin{aligned}
\mathbf{P}\{n-\nu \leqslant k-1\}= & \mathbf{P}\left\{\widetilde{S}_{n-k}^{\circ} \leqslant-\delta_{n}\right\}+o(1) \\
= & -\frac{1}{\Gamma(k)} \int_{-\infty}^{-\delta_{0}} e^{-(k-1) x} e^{-e^{-x}} d e^{-x}+o(1) \\
& -\frac{1}{\Gamma(k)} \int_{e^{\delta_{0}}}^{\infty} u^{k-1} e^{-u} d u+o(1)=\sum_{i=0}^{k-1} \frac{\lambda^{i}}{i !} e^{-\lambda}+o(1)
\end{aligned}
$$

где $\lambda=e^{\delta_{0}}$, что равносильно утверждению теоремы.

Автор признателен рецензенту за ряд ценных замечаний, способствовавших улучшению содержания работы. 


\section{СПИСОК ЛИТЕРАТУРЫ}

1. Стариев $A$. Н. О распределении размера эпидемии в одной немарковской модели. - Теория вероятн. и ее примен., 1996, т. 41, в. 4, с. 827-839.

2. Мариук Г.И. Математические методы в иммунологии. М.: Наука, 1985, 240 с.

3. Севастьянов Б. А. Ветвящиеся процессы с взаимодействием частиц. - Тезисы докладов III Международной Вильнюсской конференции по теории вероятностей и математической статистике. Т. II. Вильнюс: Институт математики и кибернетики АН ЛитССР, 1981, с. 139-140.

4. Калинкин А. В. Финальные вероятности ветвящегося процесса с взаимодействием частиц и процесс эпидемии. - Теория вероятн. и ее примен., 1998, т. 43, в. 4, c. $773-780$.

5. Ball F. A unified approach to the distribution of total size and total area under the trajectory of infectives in epidemic models. - Adv. Appl. Probab., 1986, v. 18, p. 289-310.

6. Sellke T. On the asymptotic distribution of the size of a stochastic epidemic. - J. Appl. Probab., 1983, v. 20, p. 390-394.

7. Нагаев $A . B .$, Старчев $A$. Н. Асимптотический анализ одной стохастической модели эпидемий. - Теория вероятн. и ее примен., 1970, т. 15, в. 1, с. 97-105.

8. Романовский Ю.М., Степанова Н. В., Черновский Д. С. Что такое математическая биофизика. М.: Просвещение, 1971, 136 с.

9. Феллер В. Введение в теорию вероятностей и ее приложения. Т. II. М.: Мир, 1984, $738 \mathrm{c}$.

10. Борисов И.С., Боровков А. А. Аппроксимация второго порядка для случайных ломаных в принципе инвариантности Донскера-Прохорова. - Теория вероятн. и ее примен., 1986, т. 31, в. 2, с. 225-245.

11. Startsev A.N. On approximation conditions of the distribution of the maximum sums of independent random variables. - Ann. Acad. Sci. Fenn. Ser. A I. Math., 1988, v. 13 , № 2 , p. 269-275.

12. Старчев $A . H$. Предельные теоремы для размера эпидемии в общей вероятностной модели. - Случайные процессы и статистические выводы. Ташкент: Фан, 1971, c. $60-73$.

13. Daniels H.E. The distribution of the total size of an epidemic. - Proceedings of the Fifth Berkeley Symposium on Mathematical Statistics and Probability, 1965/66. V. 4. Berkeley: Univ. of California Press, 1967, p. 281-293.

Поступила в редакцию 4.II.1999 